\title{
Velocity selection of Rydberg positronium using a curved electrostatic guide
}

\author{
A. M. Alonso, B. S. Cooper, A. Deller, L. Gurung, S. D. Hogan, and D. B. Cassidy \\ Department of Physics and Astronomy, University College London, Gower Street, London WC1E 6BT, United Kingdom
}

(Received 27 March 2017; published 19 May 2017)

\begin{abstract}
We report experiments in which a slow Rydberg positronium (Ps) beam was produced by velocity selection using a curved electrostatic quadrupole guide. Ps atoms in Rydberg-Stark states with principal quantum number $n=14$ were prepared by a two-color optical excitation process in a uniform electric field. Low-field-seeking Stark states were produced at the entrance of a $0.6-\mathrm{m}$-long quadrupole guide that includes a $45^{\circ}$ bend, and were detected at the end of the guide via their annihilation $\gamma$ radiation. The mean speed (kinetic energy) of atoms entering the guide was estimated to be $\approx 180 \mathrm{~km} \mathrm{~s}^{-1}(185 \mathrm{meV})$, whereas the mean longitudinal speed of guided atoms was measured via time of flight and found to be $\approx 90 \mathrm{~km} \mathrm{~s}^{-1}$, equivalent to a kinetic energy of $45 \mathrm{meV}$. The measured transport data are in broad agreement with Monte Carlo simulations, which are also used to establish the efficacy with which the arrangement we describe could be used to perform Ps-atom scattering measurements.
\end{abstract}

DOI: 10.1103/PhysRevA.95.053409

\section{INTRODUCTION}

Positronium (Ps), the electron-positron bound state [1], has been extensively studied since it was first produced in the laboratory in 1951 [2]. The main experimental research areas that incorporate positronium are atomic and molecular scattering [3-5], materials science [6-9], and QED tests $[10,11]$. Ps is especially interesting for fundamental physics studies as it has some unique properties compared to other atoms. For example, being composed of equal-mass leptons, it has maximal recoil effects [12], is (almost) fully described by QED [13], and can, in principle, be used to measure the Rydberg constant without hadronic complications, which may be of relevance to the proton radius puzzle [14].

In recent years the use of Surko-type positron traps [15] has had a transformative effect on Ps research [16], in particular on laser spectroscopy of Ps (e.g., [17-19]). This is because the output of a positron trap can be bunched in time [20] to a few nanoseconds (i.e., much less than the 142-ns triplet Ps ground-state lifetime [21]) and implanted into an appropriate target to form a dilute Ps gas in vacuum [22]. In this way Ps atoms can be produced with a high density and/or be addressed by pulsed lasers, facilitating new Ps research areas [23]. One example is the production of highly excited Rydberg Ps states [24] using a two-color, two-step excitation process [25,26].

Exciting Ps atoms from the ground state to Rydberg-Stark states has two beneficial consequences: First, the annihilation decay channel is essentially closed [27], which means that Ps decay rates are in effect those of fluorescence [28]. Second, Rydberg-Stark states can be produced with electric dipole moments ranging from zero to thousands of debyes for typical experimental conditions [24]. These dipole moments can be used to exert forces on atoms in Rydberg states and thus enable control of their translational motion [29].

The suggestion to exploit such forces to control the motion of atoms and molecules was first put forward by Wing [30] and Breeden and Metcalf [31]. Proof-of-principle experiments demonstrating the transverse deflection of beams of Rydberg $\mathrm{Kr}$ atoms [32] and longitudinal acceleration and deceleration of beams of $\mathrm{H}_{2}$ using time-independent electric fields were later performed by Softley and coworkers [33]. The subsequent introduction of time-dependent electric fields
[34] by Vliegen, Merkt, and coworkers permitted efficient control over the translational motion of hydrogenic [35] and nonhydrogenic [36] atoms in pulsed supersonic beams and the realization of a wide range of Rydberg atom and molecule optics elements, including mirrors [37], lenses [35], deflectors [38,39], decelerators, and traps based upon three-dimensional [40-42] and chip-based two-dimensional [43-45] electrode structures. These advances have allowed studies of the effects of blackbody-induced transitions and photoionization of Rydberg states [42,46], studies of $m$-changing dipole-dipole interactions in gases of polar Rydberg atoms and their effects on Rydberg state lifetimes [47], the preparation of long-lived high- $|m|$ (i.e., $|m| \geqslant 3$ ) Rydberg states of $\mathrm{H}_{2}$ [48,49], and new methods to study ion-molecule reactions at low temperatures [50,51]. It is only recently, however, that these methods have been applied to the Ps system [52].

Previously, we demonstrated the radial confinement of Ps atoms in Rydberg-Stark states in a straight electrostatic quadrupole guide [52]. Here we report further measurements made using a modified guide that incorporates a $45^{\circ}$ bend. This type of guiding structure has been widely used in experiments with polar molecules [53-57]. In our experiments Ps atoms with $n=14$ were transported along a curved trajectory and were detected via their annihilation radiation at the end of the guide. This arrangement has two useful properties; namely, (1) it removes the Ps beam from the positron trap axis, and (2) the curvature of the device acts as a longitudinal velocity selector. These features can, in principle, be exploited to perform experiments in which guided Ps atoms interact with other species. Furthermore, the availability of slow, long-lived, and controllable Ps beams will facilitate other experiments, such as high-resolution studies of the electronic properties of materials [58], Ps free-fall measurements [59,60], and spectroscopy of Rydberg Ps.

\section{RYDBERG ATOMS IN ELECTRIC FIELDS}

In hydrogenic atoms, states with the same $n$ but different $\ell$ values are degenerate in the absence of external fields. Consequently, even very weak electric or magnetic fields, both of which are invariably present in our experiments, can lead 


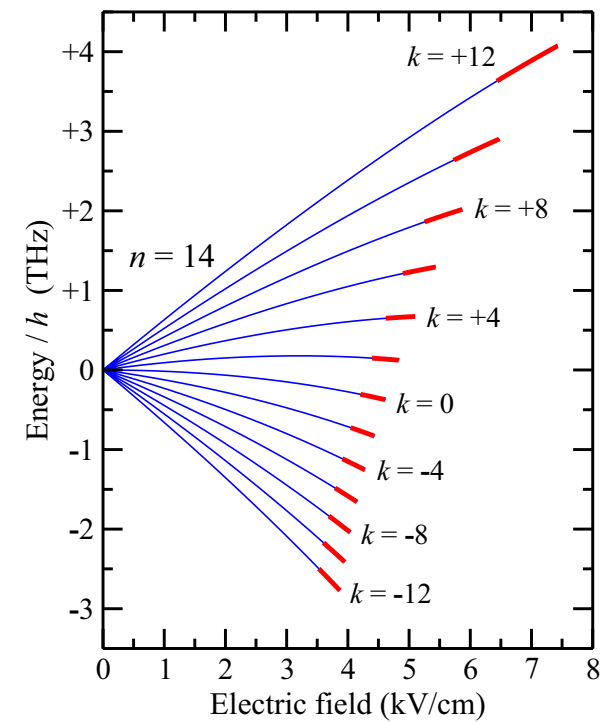

FIG. 1. Stark structure at $n=14$ in Ps. Each Stark state, labeled with the index $k$ (see text for details), is displayed for fields in which the ionization rate is $<10^{10} \mathrm{~s}^{-1}$. The thicker sections of each curve indicate the fields for which the ionization rate ranges from $\geqslant 10^{8}$ to $\leqslant 10^{10} \mathrm{~s}^{-1}$.

to complete $\ell$ mixing within an $n$ manifold [61]; in the latter case this is due to motional Stark effects [62]. Under these conditions it is often more convenient to consider the atomic structure in terms of hydrogenic Stark states. If the Schrödinger equation is solved in parabolic coordinates, the resulting eigenstates are labeled according to their parabolic quantum numbers $n_{1}$ and $n_{2}$, and the Stark states may then be characterized using the index $k=n_{1}-n_{2}$ [24]. For each value of $n$ and the azimuthal quantum number $m$, the allowed values of $k$ range from $-(n-|m|-1)$ to $+(n-|m|-1)$ in intervals of 2 .

In an electric field $\vec{F}=\left(0,0, F_{z}\right)$, the energy shifts of these Stark states can be expressed analytically to second order as [63]

$$
\begin{aligned}
E_{\text {Stark }}= & \frac{3}{2} n k e a_{\mathrm{Ps}} F_{z}+\cdots \\
& -\frac{1}{16} n^{4}\left(17 n^{2}-3 k^{2}-9 m^{2}+19\right) \frac{e^{2} a_{\mathrm{Ps}}^{2}}{2 h c R_{\mathrm{Ps}}} F_{z}^{2},
\end{aligned}
$$

where $e$ is the charge of the electron, and $a_{\mathrm{Ps}}$ and $R_{\mathrm{Ps}}$ are the Bohr radius and Rydberg constant corrected for the reduced mass of Ps, respectively. Stark shifts for $n=14$ Ps atoms with $m=1$, calculated using this expression, are shown in Fig. 1 [64].

Since the energy shift of an electric dipole in an electric field $\vec{F}$ is

$$
E_{\text {Stark }}=-\vec{\mu}_{\text {elec }} \cdot \vec{F}
$$

comparison with Eq. (1) indicates that, to first order, an electric dipole moment $\vec{\mu}_{\text {elec }}$ can be associated with each RydbergStark state such that [24]

$$
\vec{\mu}_{\text {elec }}=-\frac{3}{2} n k e a_{\mathrm{Ps}} .
$$

These large static electric dipole moments allow forces to be exerted on samples in these states using inhomogeneous electric fields [29]. In a spatially inhomogeneous electric field the resulting force is

$$
\vec{f}=\nabla\left(\vec{\mu}_{\text {elec }} \cdot \vec{F}\right) .
$$

The magnitude of this force therefore depends directly on the gradient of the electric field. In exploiting such forces to control the translational motion of Rydberg atoms the greatest accelerations that can be achieved using time-independent electric potentials are limited by field ionization of the Rydberg states. The tunnel ionization rate $\Gamma_{n n_{1} n_{2} m}\left(F_{z}\right)$ for a RydbergStark state in an electric field is given by [63]

$$
\begin{aligned}
\Gamma_{n n_{1} n_{2} m}\left(F_{z}\right) \\
=\frac{2 h c R_{\mathrm{Ps}}}{\hbar} \frac{(4 C)^{2 n_{2}+m+1}}{n^{3} n_{2} !\left(n_{2}+m\right) !} \\
\quad \times \exp \left[-\frac{2}{3} C-\frac{1}{4} n^{3} \frac{e a_{\mathrm{Ps}} F_{z}}{2 h c R_{\mathrm{Ps}}}\left(34 n_{2}^{2}+34 n_{2} m\right.\right. \\
\left.\left.\quad+46 n_{2}+7 m^{2}+23 m+\frac{53}{3}\right)\right]
\end{aligned}
$$

where

$$
C=\frac{1}{e a_{\mathrm{Ps}} \sqrt{2 h c R_{\mathrm{Ps}}}} \frac{\left(-2 E_{n n_{1} n_{2} m}\right)^{3 / 2}}{F_{z}}
$$

and $E_{n n_{1} n_{2} m}=-\left[h c R_{\mathrm{Ps}} /\left(n^{2}\right)\right]+E_{\mathrm{Stark}}$ is the total energy of the state with respect to the ionization limit in the presence of the electric field. The Stark map presented in Fig. 1 is displayed for fields up to those in which the ionization rate exceeds $10^{10} \mathrm{~s}^{-1}$. The thicker sections of each curve indicate the fields for which the ionization rate ranges from $\geqslant 10^{8}$ to $\leqslant 10^{10} \mathrm{~s}^{-1}$.

\section{EXPERIMENTAL METHODS}

The apparatus [22] and detection methods [28,65] used in this work are similar to those used previously, and only a short overview will be given here. A pulsed positron beam was produced using a two-stage Surko-type buffer-gas positron trap [16]. The output of the trap $\left(\approx 10^{5}\right.$ positrons, $\Delta t \approx 4 \mathrm{~ns}$ ) was implanted into a mesoporous silica film $[66,67]$ which produced Ps atoms in vacuum. These atoms were optically excited using two dye lasers: an ultraviolet (UV) laser $(\approx 500 \mu \mathrm{J}, \Delta v=85 \mathrm{GHz}, \lambda=243.0 \mathrm{~nm})$ was used to drive $1^{3} S \rightarrow 2^{3} P$ transitions and an infrared (IR) laser $(\approx 8 \mathrm{~mJ}, \Delta v=5 \mathrm{GHz}, \lambda \approx 744 \mathrm{~nm})$ was used to drive $2^{3} P \rightarrow 14{ }^{3} S / 14^{3} D$ transitions. This excitation scheme couples ground-state atoms to excited states with $S$ or $D$ character. The UV and IR laser light was linearly polarized in the vertical direction, which means that Stark states were produced predominantly with azimuthal quantum number $m=1$ and even values of $k$.

As shown in Fig. 2, Ps atoms were produced in an excitation region between two electrodes (target and grid). This made it possible to prepare Rydberg states in a uniform electric field and thus to address specific parts of the Stark manifold [68]. By tuning the IR laser to shorter wavelengths we were able to predominantly excite Stark states with $k=+12$ and 

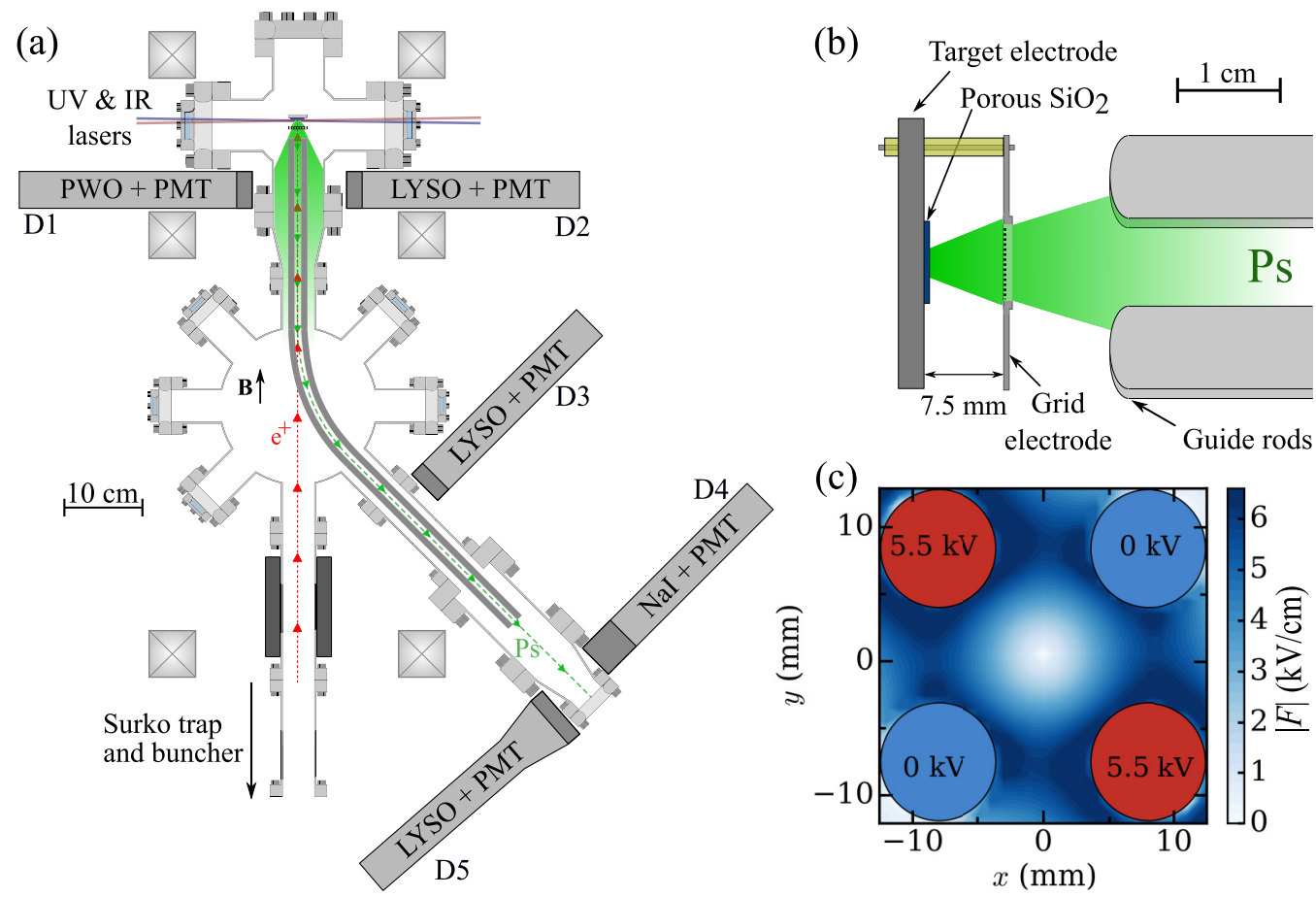

FIG. 2. (a) Schematic representation of the experimental apparatus containing the curved guide. The positions of the five $\gamma$-ray detectors used in the experiment are indicated (see text for details). D1 and D2 are used to monitor Ps atoms in or near the excitation region via lifetime spectroscopy, whereas D3, D4, and D5 are used to generate single-event TOF spectra. (b) Close-up of the excitation region indicating the Ps formation target and excitation region and the position of the quadrupole guide rods relative to the grid electrode and (c) electric-field map within the guide with $5.5 \mathrm{kV}$ applied to one pair of rods.

dipole moments of $1280 \mathrm{D}$ (see Sec. II). The geometry of the arrangement is such that some Rydberg atoms are able to enter the guide, whereas others collide with the guide rods, the vacuum chamber, or the grid electrode. The quadrupole guide is constructed from four cylindrical electrodes $8 \mathrm{~mm}$ in diameter and separated from each other by $7 \mathrm{~mm}$. The total length of the quadrupole is $600 \mathrm{~mm}$, and there is a $45^{\circ}$ bend, as indicated in Fig. 2(a). The guide is operated with one pair of rods grounded and the other pair biased up to $5.5 \mathrm{kV}$. The guide field is turned off before the positron beam implantation and is switched on $\approx 50 \mathrm{~ns}$ after the positrons enter the silica target, which is before any Ps atoms can exit the excitation region. Some of the Ps atoms in states with positive Stark shifts [low-field-seeking (lfs) states] that enter the guide structure are deflected away from the electrodes by the inhomogeneous electric fields and thus stay in the device, whereas atoms in states with negative Stark shifts [high-fieldseeking (hfs) states] would be deflected out of the guide or collide with the rods and be lost.

Five $\gamma$-ray detectors were used in the experiment to observe radiation resulting from Ps-electrode or wall collisions, from self-annihilation, or from the annihilation of positrons liberated via field ionization or photoionization. The locations of the detectors are indicated in Fig. 2. D1 incorporates a lead tungstate (PWO) scintillator [69] and is used to monitor the positron beam time width and Ps formation efficiency. D2, D3, and D5 use lutetium-yttrium oxyorthosilicate (LYSO) scintillators [65], and D4 contains a sodium iodide (NaI) scintillator. Detectors located close to the Ps formation target (D1 and D2) are irradiated by many near-simultaneous $\gamma$ rays following the positron beam implantation. In this case we record single-shot lifetime spectra [70] and the signal parameter $S_{\gamma}$, which is proportional to the number of atoms excited by the lasers $[22,65]$. Detectors that are farther away from the Ps production region (D3-D5) are used to count individual annihilation events, from which we construct timeof-flight (TOF) spectra using the procedures described in Ref. [28].

\section{RESULTS AND DISCUSSION}

Rydberg Ps atoms were prepared both with and without a uniform electric field present. The corresponding line shapes, measured using D2, for the $2^{3} P \rightarrow 14^{3} S / 14^{3} D$ transitions are shown in Fig. 3(a). The Stark-broadened transition allows us to tune the laser wavelength to preferentially excite lfs states [68]. This is not possible if the excitation is performed with zero applied field because we have insufficient spectral resolution (owing primarily to the laser bandwidth and Doppler broadening) to address only the part of the Stark manifold containing the desired lfs states. These states still exist even when no field is applied as a result of the magnetic field and stray electric fields in the excitation region [28].

Rydberg atoms that enter the quadrupole guide structure and are transported to the end are detected by D4 and D5. These detectors are sufficiently far away from the Ps production region that they do not observe simultaneous events, and a single-event counting procedure is therefore used [28]. The total event rate recorded during the line-shape measurements is shown in Fig. 3(b). Here we see that when lfs states 


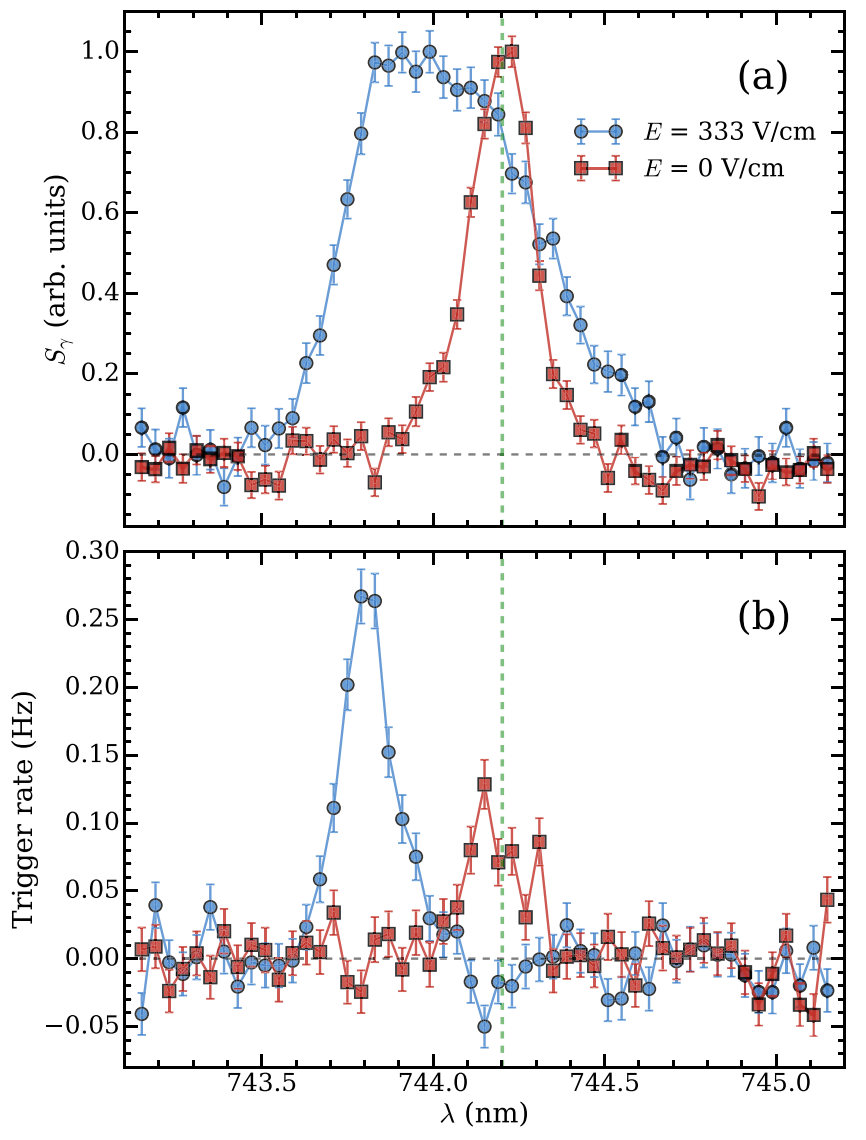

FIG. 3. (a) Line shapes for the $2{ }^{3} P_{J} \rightarrow 14{ }^{3} S / 14^{3} D$ transitions measured by $\mathrm{D} 2$ with and without a $333 \mathrm{~V} / \mathrm{cm}$ electric field applied in the excitation region. The asymmetric line shape measured with the electric field applied is due to deflection of atoms towards or away from D2. The data have been normalized to the peak amplitude. (b) Total count rates for guided atoms as a function of the IR laser wavelength in the corresponding electric fields, measured by D4/5. The dashed vertical line represents the expected zero-field resonant wavelength, and the voltage applied to the guide was $5.5 \mathrm{kV}$.

are preferentially prepared, the event rate is much higher and occurs at a wavelength that is downshifted from the resonant wavelength. These data show conclusively that it is more efficient to broaden the line and then tune the laser to the lfs side of the spectrum, which is to be expected since we will not then produce any hfs states, which cannot be guided. This procedure will mostly result in the production of Rydberg-Stark states with values of the parabolic quantum number $k=+12$ and dipole moments of $1280 \mathrm{D}$ (see Sec. II). The maximum electric-field strength encountered in the guide is $\approx 7 \mathrm{kV} \mathrm{cm}^{-1}$, which would result in field ionization rates of the $k=+12$ Stark states of $\approx 10^{9} \mathrm{~s}^{-1}$ (see Fig. 1). However, simulations indicate that the Ps atoms do not spend much time in these field extrema.

TOF spectra of guided atoms recorded near the bend (by D3, see Fig. 2) and at the end of the guide (by D4/5) for different applied electric potentials are shown in Fig. 4. These spectra have been background subtracted, where the background corresponds to the case where no IR laser light is present. The data show that increasing the quadrupole field

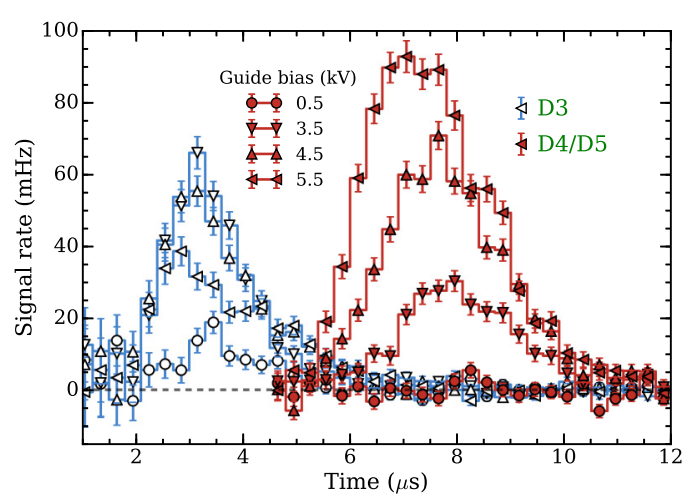

FIG. 4. TOF data measured using D3 and D4/5 for guide voltages of $0.5,3.5,4.5$, and $5.5 \mathrm{kV}$, as indicated in the legend. Additional spectra (not shown) were recorded for guide voltages of 1.5, 2.5, and $5.0 \mathrm{kV}$ and lfs Ps atoms with $n=14$. Each curve was acquired in $4 \mathrm{~h}$.

strength increases the overall guiding efficiency by capturing more of the Ps atoms. The count rate measured by D3 has a maximum value at intermediate guiding fields because D3 will predominantly detect atoms that are guided in the straight section but are not transported around the bend.

Ps emission from mesoporous materials has been widely studied [71-80] since they may be used as convenient and robust sources of Ps atoms. The mesoporous silica film used in the present work contains randomly distributed, interconnected pores with diameters of $\approx 5 \mathrm{~nm}$. The mean Ps energy as a function of the positron implantation energy has been studied previously using TOF methods [79]; for positron implantation energies above a few $\mathrm{keV}$, Ps is emitted with mean energies $\leqslant 100 \mathrm{meV}$. The angular distribution of Ps emitted from mesoporous films is, however, less well understood. Mean Ps energies measured by TOF [79] or Doppler [74] methods include some component due to the angular distributions, and we cannot therefore accurately describe the initial Ps distribution as accepted into the guiding structures. Also the relative position and timing of the excitation lasers with the Ps atoms affect which part of the underlying ground-state distribution will be excited to Rydberg levels [79].

We may evaluate the degree of velocity selection imposed by the curved guide by considering velocity distributions measured with and without guiding, as shown in Fig. 5. These distributions were derived from measured TOF distributions, with the data rebinned into equal-sized $\left(10 \mathrm{~km} \mathrm{~s}^{-1}\right)$ bins. The "no-guide" data were recorded using $n=20$ Ps atoms, measured $1.2 \mathrm{~m}$ away from the Ps production region [28] and with a positron beam energy of $2 \mathrm{keV}$. In this configuration only atoms emitted in a small $\left(1.5^{\circ}\right)$ cone were detected, and therefore atoms with the largest (smallest) longitudinal (radial) speeds were selected. A mean Ps speed of $115 \mathrm{~km} \mathrm{~s}^{-1}$ was measured near the Ps source region [79], while $\approx 225 \mathrm{~km} \mathrm{~s}^{-1}$ was measured $1.2 \mathrm{~m}$ away, as shown in Fig. 5. This spectrum represents an upper limit to the possible initial Ps distribution as accepted into the guiding structures.

Ps atoms transported by the straight guide are radially trapped [52]; this introduces some longitudinal velocity selection owing to the correlation between the longitudinal 


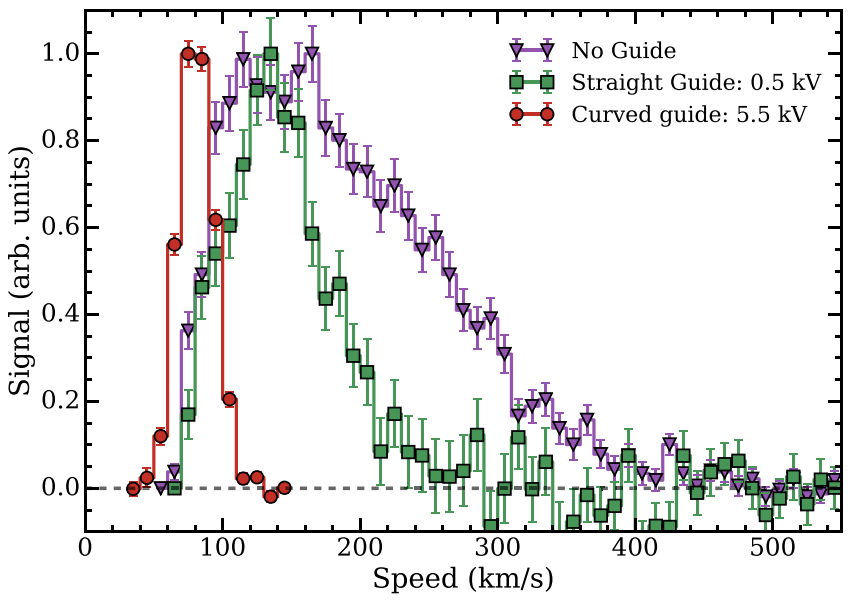

FIG. 5. Longitudinal velocity distributions as derived from TOF measurements obtained with no guide (triangles) [28], with a straight guide (squares) [52], and with the curved guide (circles). The electric potential applied to the guide rods is indicated in the legend. The curved-guide data are derived from the data shown in Fig. 4. TOF spectra were rebinned into $10 \mathrm{~km} \mathrm{~s}^{-1}$ steps to generate the velocity distributions.

and transverse velocity components [79]. The straight-guide distribution shown in Fig. 5 was obtained using a relatively low guiding field, which therefore selected slower atoms. This spectrum indicates a mean speed of $137 \mathrm{~km} \mathrm{~s}^{-1}$ and represents a lower limit to the possible initial Ps distribution at the guide entrance.

Thus, as a rough approximation, we can expect a representation of the initial Ps velocity distribution entering the curved guide to fall somewhere between the unguided and straight guide distributions shown in Fig. 5. We estimate that the mean Ps speed entering the curved guide was $\approx 180 \mathrm{~km} \mathrm{~s}^{-1}$, whereas the mean speed of atoms leaving the guide is $\approx 90 \mathrm{~km} \mathrm{~s}^{-1}$. The absolute number of atoms detected in each case depends on several factors, such as guide efficiency, beam collimation, Ps-guide alignment, and detector solid angle. These factors are not necessarily the same for all measurements, and the unguided case is, of course, intrinsically much less efficient. The distributions have been normalized to the peak amplitude to facilitate comparison of the mean speeds, but the transport and detection efficiencies will be different in each case.

\section{SIMULATIONS}

Simulations of the motion of Ps atoms in Rydberg-Stark states in the electrostatic quadrupole field were carried out to help us understand the experimental data and also to examine the possibility of performing scattering measurements using guided Rydberg Ps. The equations of motion of Ps atoms in a quadrupole electric field were solved to find the Ps trajectories using the fourth-order Runge-Kutta algorithm, with the acceleration of each atom given by

$$
\vec{a}=\frac{\mu_{\mathrm{elec}}}{2 m_{e}} \nabla|\vec{F}| \text {. }
$$

The electric-field gradients were obtained using finite-element calculations of the electric-field distribution within the guide as shown in Fig. 6.

The trajectory calculation was seeded using a Monte Carlo simulation of the initial Rydberg Ps velocity distribution. Ground-state Ps were modeled as being emitted from the converter with a thermal speed distribution $(T=1200 \mathrm{~K})$ and cosine angular distribution. Ps produced from porous silica films is not expected to exhibit a thermal distribution, but previous measurements [79] have shown that the actual Ps distributions can be approximated with reasonable accuracy in this way using an arbitrary temperature value. The subset of Ps atoms that could be laser excited to Rydberg levels was found using a simple threshold condition for the temporal, spatial, and spectral overlap with a $\Delta t=5 \mathrm{~ns}$ pulsed laser field. The spatial overlap was based on a $4 \times 8 \mathrm{~mm}$ laser beam. The spectral overlap was determined by the laser bandwidth, $\Delta v=100 \mathrm{GHz}$, and the Doppler shift of each atom according to its velocity in the direction parallel to the propagation of the laser $v_{x}$.

The Ps atom trajectories were calculated taking into account the electric field experienced by each atom and the corresponding ionization rate (see Sec. II) as well as the
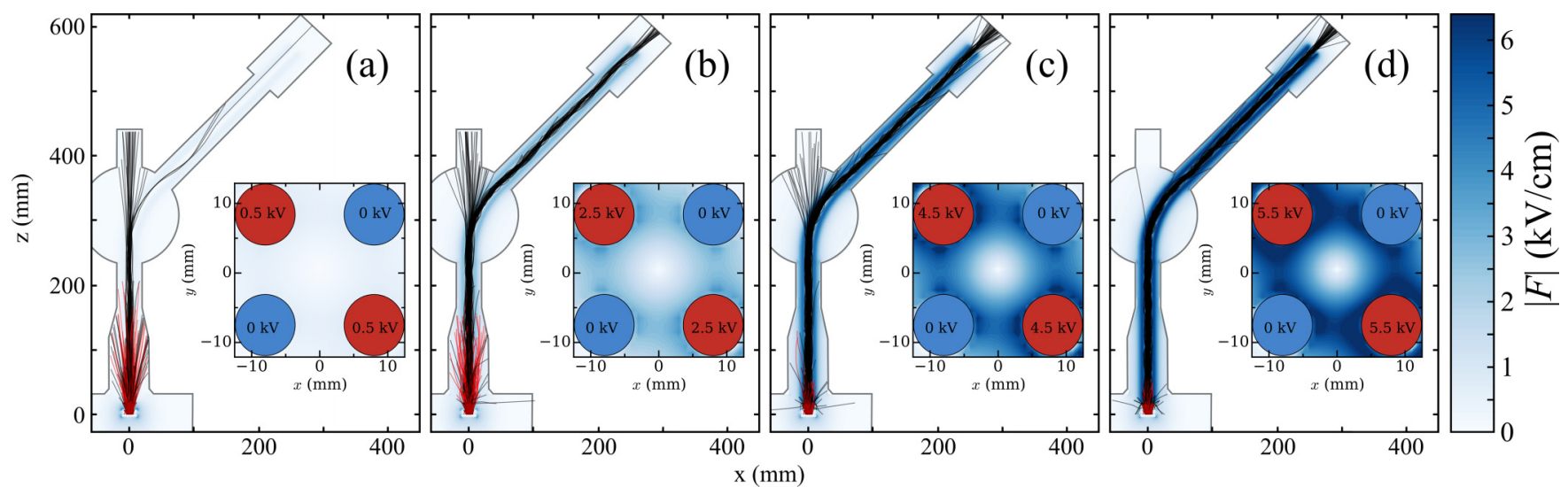

FIG. 6. Simulated trajectories for guide biases of (a) 0.5 , (b) 2.5 , (c) 4.5 , and (d) $5.5 \mathrm{kV}$. The black lines represent atoms in the low-fieldseeking state with $k=+12$, whereas the lighter red lines represent atoms in the high-field-seeking state with $k=12$. Also shown in each panel is an inset of the electric-field strength for each configuration and an outline of the chamber walls representing the ground plane of the electric field. 


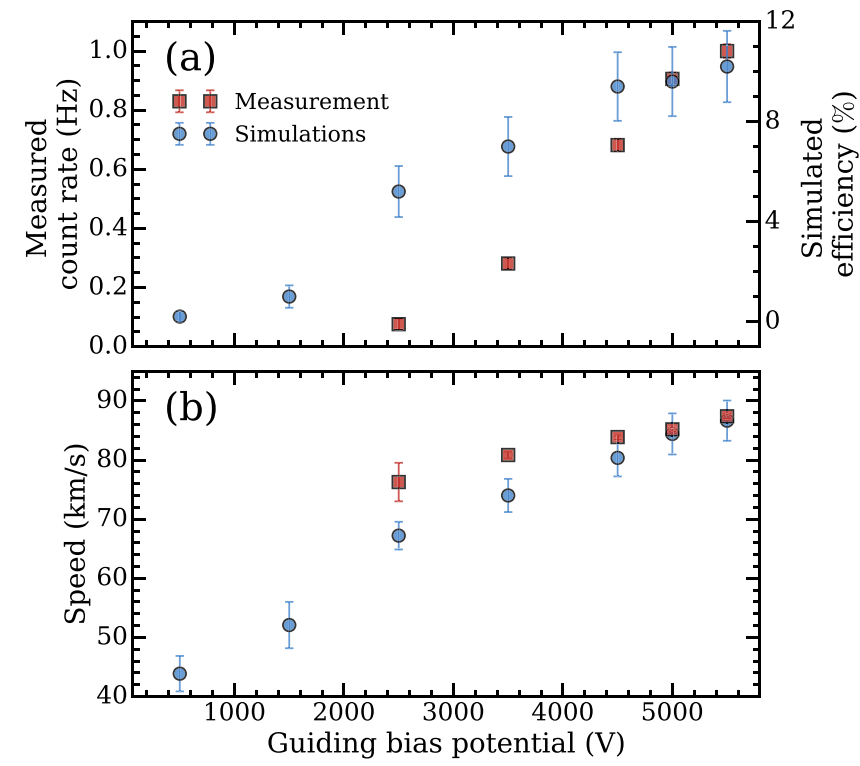

FIG. 7. (a) Measured count rate and simulated guiding efficiency and (b) mean Ps velocities derived from the TOF data and simulations for different voltages applied to the quadrupole rods.

Ps fluorescence lifetimes. The latter were obtained from the calculated values described in Ref. [28]. If an atom decayed, was field ionized, or collided with chamber walls, the grid, the target, or the guide electrodes, the trajectory was terminated. The Monte Carlo simulations were run using 1000 particles, and the results are shown in Fig. 6 for different guiding fields.

For our initial velocity distributions and the maximum guide voltages used in our experiments the simulations indicate that $n=14$ is the optimal guiding state based on the guide efficiency and loss mechanisms (decay and ionization). The data in Fig. 6(d) show that at these fields Ps atoms that are not guided will be ionized before leaving the guide structure. The simulations indicate that higher $n$ states can be guided in lower fields, although this may only be possible if the excitation region field is turned off before the Ps atoms exit through the grid electrode [81] to prevent field ionization [68].

Figure 7 shows a comparison of the measured event rate and calculated guiding efficiency, as well as the measured and calculated mean Ps speeds. We find a broad agreement over the range for which measurements were performed. The TOF distributions were fitted to Gaussian functions in order to estimate the mean Ps speeds, and we find reasonable agreement between the measurements and simulations. These data indicate that by adjusting the guide field, the mean speed of atoms can be controlled over a small range (approximately $75-90 \mathrm{~km} \mathrm{~s}^{-1}$ ). However, this variation is small compared to the width of the velocity distribution and is accompanied by a large reduction in the guiding efficiency.

The measured TOF distributions can be used to evaluate the efficacy with which our arrangement could be used to observe the formation of positron-atom bound states. To do this the attenuation of the measured Ps TOF distributions was generically simulated by including an interaction cross section and assuming an atom was removed from the system if a scattering event occurred. The cross sections used were

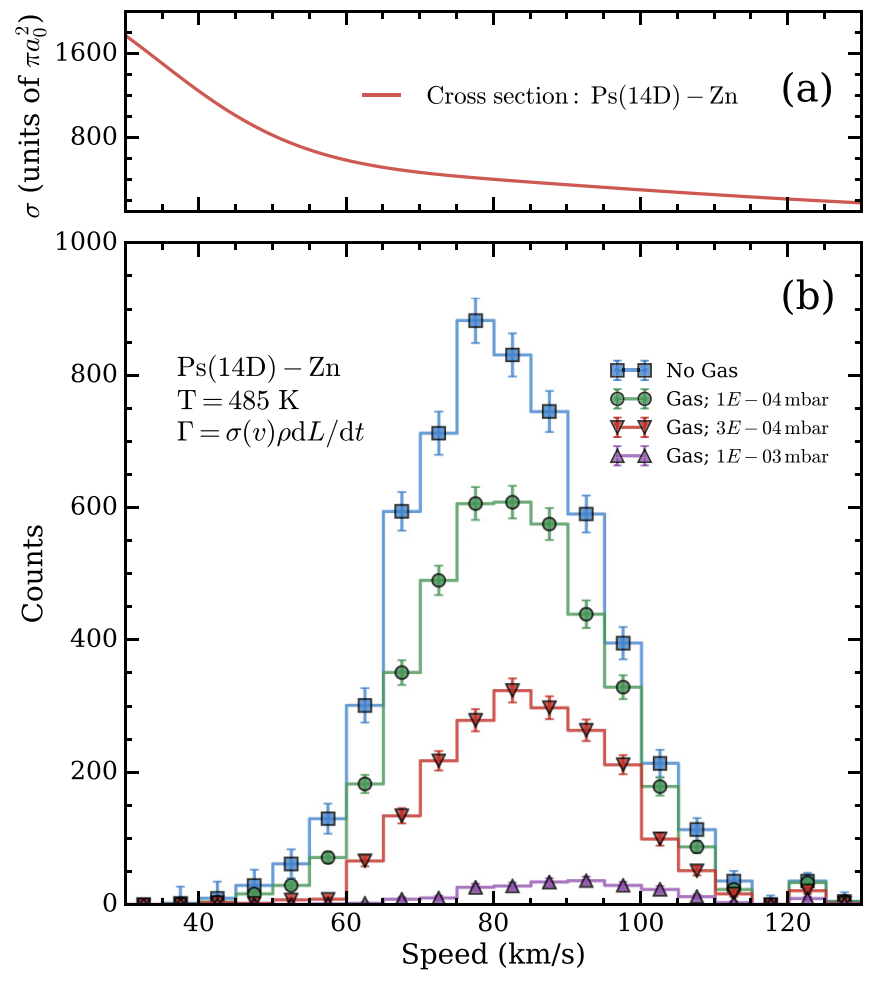

FIG. 8. (a) Calculated cross section $\sigma$ for positron-atom boundstate formation in collisions of $n=14$ Ps atoms with $\mathrm{Zn}$ atoms and (b) simulated data indicating how the observed velocity distribution would be attenuated by Ps charge-exchange reactions with $\mathrm{Zn}$ at the indicated pressures. The measured TOF distribution has been rebinned into $5 \mathrm{~km} \mathrm{~s}^{-1}$ steps to generate the velocity distribution.

those calculated for positrons binding to $\mathrm{Zn}$ atoms [82] and are shown in Fig. 8(a). The simulated attenuation of the Ps distribution is shown in Fig. 8(b) for different $\mathrm{Zn}$ pressures at a temperature of $485 \mathrm{~K}$. The trajectories shown in Fig. 6(d) were used as the basis for Ps-atom interactions in a 3.5-cm-long scattering region that encompassed the entire radial extent of the beam. The velocity of each particle was obtained from a weighted distribution based on the measured data shown in Fig. 4, and the interaction rate per particle in the simulation $\Gamma$ was given by

$$
\Gamma=\sigma(v) \rho d L / d t
$$

where $\rho$ is the target species number density, $d L$ is the distance traveled in each time step $d t$, and $\sigma(v)$ is the relevant cross section as a function of speed, obtained from Ref. [82] and shown in Fig. 8(a). For each particle whether an event had occurred or not was determined by comparing the calculated probability with a randomly generated number between 0 and 1. The simulations were run with 5865 particles for each pressure shown in Fig. 8. We note that the cross sections used in the simulations were calculated for Ps Rydberg levels with specific angular momentum $\ell$ values (in this case, $d$ states), whereas experimentally, we expect to produce only Rydberg-Stark states (i.e., states that are strongly $\ell$ mixed) [28]. However, test calculations designed to estimate the likely cross section $\ell$ dependence indicate that below energies of $1 \mathrm{eV}$ it is relatively weak [82]. Nevertheless, our determination of 
possible positron-atom bound-state production rates should be considered only as an order of magnitude estimate.

Figure 8 shows the simulated attenuation of the Ps distribution for different $\mathrm{Zn}$ gas pressures in the scattering region. These data indicate that a clearly discernible signal could be observed but that it would require $\mathrm{Zn}$ pressures close to $1 \times 10^{-4}$ mbar, unless slower Ps atoms can be employed. Such pressures can be achieved by heating $\mathrm{Zn}$ to temperatures $\gtrsim 500 \mathrm{~K}$ [83]. Although there are some experimental challenges associated with producing hot metal vapors, it can be achieved by integrating a hot cell into our system (e.g., [84]), possibly via a magnetic guide [85]. If this can be successfully accomplished with $\mathrm{Zn}$, then our simulations indicate that a measurement of positron-atom binding is, in principle, feasible using our existing Ps source.

\section{CONCLUSIONS}

We have demonstrated velocity selection via the operation of a curved electrostatic quadrupole guide using RydbergStark states of Ps with $n=14$. The mean speed of the unguided Ps distribution was estimated to be approximately $180 \mathrm{~km} \mathrm{~s}^{-1}$, whereas for the guided atoms $\approx 90 \mathrm{~km} \mathrm{~s}^{-1}$ was measured. From a practical point of view the curved guide is very convenient in that one can remove the Ps beam from the trap axis, allowing for improved separation of the detection and production regions. A rotated Ps formation target can also be used to achieve this goal (e.g., [86]) but at the cost of increasing the effective positron beam-spot size and hence the spatial extent of the Ps source. Alternatively, one can bend the incident positron beam using magnetic fields (e.g., [28]), although this can make the beam alignment more complicated. Moreover, both of these methods retain a direct line of sight between the excitation and detection regions, which for some experimental arrangements could be undesirable; for example, scattered laser light may reach the interaction region and interact with sensitive microchannel plate detectors [87], or the Ps formation target may become contaminated by scattering-target gases.

Ps production methods generally involve the interaction of energetic positrons with solid [9,73,88-92] or gaseous $[5,93,94]$ targets. Depending on the formation mechanism, Ps atoms may be created with energies ranging from many $\mathrm{eV}$ (e.g., [90]) to thermal energies [95,96] (i.e., $\approx 25 \mathrm{meV}$ at $300 \mathrm{~K})$. Some methods have been developed to produce colder Ps but are either inefficient [97] or rely on unstable surface conditions [98]. In the absence of new sources of cold Ps atoms, one can resort to velocity selection, as we have demonstrated here. However, in general this is not the preferred option for Ps experiments since the number of atoms available is already extremely low. This is in contrast to experiments using ordinary gases, for which the total number of atoms or molecules is extremely high (compare with Avogadro's number), and so even extreme velocity selection can result in a high particle flux. For example, curved electrostatic guides have been successfully employed to generate useful beams of cold polar molecules from a thermal distribution by velocity selection [53-57]. In the first demonstration of this technique, Rangwala and coworkers were able to obtain $10^{9} \mathrm{H}_{2} \mathrm{CO}$ molecules/s with a longitudinal temperature of a few kelvin from a room-temperature reservoir [53]. For the most part, such extreme losses cannot be tolerated in Ps experiments; however, in certain cases and with less stringent velocity selection, some experiments can benefit from using this approach.

We have investigated the feasibility of producing positronatom bound states. There is a large amount of theoretical evidence supporting the existence of such states [99-101], but they have not yet been experimentally observed [102], in contrast to positron-molecule binding, which has been extensively studied [103]. Positron interactions with negative ions $\left(e^{+}+A^{-} \rightarrow e^{+} A+e^{-}\right)$[104] and photon-assisted recombination $\left(e^{+}+A+v \rightarrow e^{+} A\right)$ [105] have been suggested as methods to produce positron-atom bound states. However, as discussed in Ref. [82], producing these systems via Rydberg Ps collisions with atoms ( $\mathrm{Ps}^{*}+A \rightarrow e^{+} A+e^{-}$) offers some advantages: for example, the cross sections are relatively large and the $e^{+} A$ binding energies can be probed by controlling the principal quantum number of the Ps Rydberg states. As indicated in Fig. 8, the Ps source we have is, in principle, suitable for these experiments.

In addition to producing positron-atom bound systems, the curved-guide arrangement could also be used to measure Ps* scattering rates from other atoms and molecules. Collisions between Rydberg atoms and neutral species have been studied for decades (e.g., [106]) and are of interest in the context of low-energy electron scattering [107]. This is because the electron is so weakly bound in a highly excited Rydberg state that in a collision it may have a stronger interaction with the target species than with its own ion core and hence behave as a free electron with an energy dictated by its orbital motion. This effect has also been observed in total scattering measurements between ground-state Ps atoms with several atomic and molecular species [108,109]. In these experiments Ps beam energies in the 10-100 eV range are used, which is higher than the Ps binding energy $(6.8 \mathrm{eV})$. Thus, much as with the Rydberg atoms, Ps-atom or -molecule interactions can then be approximated by the superposition of outgoing waves generated by the individual projectiles [110]. Since positron scattering is generally weaker than electron scattering in this energy regime [4], the observed total scattering cross sections are therefore more similar to those found in electron interactions [111]. It would be informative to perform similar measurements using Rydberg Ps atoms in different Stark states to control the orientation of the electron and positron wave functions relative to the projectile axis.

It may also be possible to study charge-exchange reactions with charged targets, for example, hydrogen formation following Ps interactions with a beam of protons [112], or with protons held in a trap $\left(\mathrm{Ps}^{*}+p \rightarrow \mathrm{H}+e^{+}\right)$. These experiments would require some modifications to the apparatus (we note that electrostatic guides have been integrated with ion traps [113] and Stark decelerators [114] before) but could otherwise be performed in much the same way as the scattering experiments described above. Such measurements are of interest in the context of antihydrogen formation via Ps-antiproton collisions [115], which is currently being pursued by several research groups at CERN [116,117]. Verifying the (anti)hydrogen production rate as a function of Ps speed and principal quantum number $n$ for this reaction would be directly relevant to antihydrogen research. Under some conditions, the expected cross sections for this process 
$[118,119]$ are orders of magnitude larger than those calculated for positron-atom production [82].

The output of the curved guide may also be used to perform spectroscopy of Rydberg Ps energy levels. It provides a relatively slow and partially collimated beam that can be transmitted through a well-characterized interaction region. This could be a microwave guide or an optical cavity, where external fields could be accurately measured and controlled (e.g., [120]). In this way it would be possible to drive transitions between nearby Rydberg levels (e.g., $n=25 \rightarrow 27$ transitions with $375 \mathrm{GHz}$ radiation) or to optically pump atoms from Rydberg levels down to $n=2$ using IR light from a c.w. laser. In either case one could obtain a measurement of the Rydberg constant that is unaffected by proton radius corrections [121] and hence could potentially help resolve the ongoing proton radius puzzle [14]. Such measurements would require several additional components to reach a useful level of precision, including extensive field characterization and cancellation, but the availability of slower atoms with some degree of collimation, as we have demonstrated here, is an important step in this direction. In future work we will attempt to couple this methodology with other Stark-manipulation techniques, such as deceleration, focusing, and trapping [29].

\section{ACKNOWLEDGMENTS}

The authors gratefully acknowledge L. Liszkay for providing silica samples and A. R. Swann and G. F. Gribakin for helpful discussions and for providing cross-section data files. This work was supported by UCL through its Impact Studentship Programme and was funded in part by the ERC (Grant No. CIG 630119) and the EPSRC (Grant No. EP/K028774/1).
[1] J. A. Wheeler, Polyelectrons, Ann. N. Y. Acad. Sci. 48, 219 (1946).

[2] M. Deutsch, Evidence for the formation of positronium in gases, Phys. Rev. 82, 455 (1951).

[3] M. Charlton and J. W. Humberston, Positron Physics, Cambridge Monographs on Atomic, Molecular and Chemical Physics Vol. 2 (Cambridge University Press, Cambridge, 2001).

[4] C. M. Surko, G. F. Gribakin, and S. J. Buckman, Low-energy positron interactions with atoms and molecules, J. Phys. B 38, R57 (2005).

[5] G. Laricchia, S. Armitage, Á. Kövér, and D. J. Murtagh, Ionizing collisions by positrons and positronium impact on the inert atoms, Adv. At. Mol. Opt. Phys. 56, 1 (2008).

[6] A. P. Mills, Jr., L. Pfeiffer, and P. M. Platzman, Positronium Velocity Spectroscopy of the Electronic Density of States at a Metal Surface, Phys. Rev. Lett. 51, 1085 (1983).

[7] D. W. Gidley, W. E. Frieze, T. L. Dull, A. F. Yee, E. T. Ryan, and H.-M. Ho, Positronium annihilation in mesoporous thin films, Phys. Rev. B 60, R5157 (1999).

[8] D. B. Cassidy, K. T. Yokoyama, S. H. M. Deng, D. L. Griscom, H. Miyadera, H. W. K. Tom, C. M. Varma, and A. P. Mills, Positronium as a probe of transient paramagnetic centers in $a-\mathrm{SiO}_{2}$, Phys. Rev. B 75, 085415 (2007).

[9] A. C. L. Jones, H. J. Goldman, Q. Zhai, P. Feng, H. W. K. Tom, and A. P. Mills, Monoenergetic Positronium Emission from Metal-Organic Framework Crystals, Phys. Rev. Lett. 114, 153201 (2015).

[10] S. Berko and H. N. Pendleton, Positronium, Annu. Rev. Nucl. Part. Sci. 30, 543 (1980).

[11] A. Rich, Recent experimental advances in positronium research, Rev. Mod. Phys. 53, 127 (1981).

[12] G. T. Bodwin, D. R. Yennie, and M. A. Gregorio, Recoil effects in the hyperfine structure of QED bound states, Rev. Mod. Phys. 57, 723 (1985).

[13] S. G. Karshenboim, Precision study of positronium: Testing bound state QED theory, Int. J. Mod. Phys. A 19, 3879 (2004).

[14] R. Pohl, R. Gilman, G. A. Miller, and K. Pachucki, Muonic hydrogen and the proton radius puzzle, Annu. Rev. Nucl. Part. Sci. 63, 175 (2013).
[15] C. M. Surko, M. Leventhal, and A. Passner, Positron Plasma in the Laboratory, Phys. Rev. Lett. 62, 901 (1989).

[16] J. R. Danielson, D. H. E. Dubin, R. G. Greaves, and C. M. Surko, Plasma and trap-based techniques for science with positrons, Rev. Mod. Phys. 87, 247 (2015).

[17] D. B. Cassidy, M. W. J. Bromley, L. C. Cota, T. H. Hisakado, H. W. K. Tom, and A. P. Mills, Jr., Cavity Induced Shift and Narrowing of the Positronium Lyman- $\alpha$ Transition, Phys. Rev. Lett. 106, 023401 (2011).

[18] D. B. Cassidy, T. H. Hisakado, H. W. K. Tom, and A. P. Mills, Jr., Laser Excitation of Positronium in the Paschen-Back Regime, Phys. Rev. Lett. 106, 173401 (2011).

[19] D. B. Cassidy, T. H. Hisakado, H. W. K. Tom, and A. P. Mills, Positronium Hyperfine Interval Measured via Saturated Absorption Spectroscopy, Phys. Rev. Lett. 109, 073401 (2012).

[20] A. P. Mills, Jr., Time bunching of slow positrons for annihilation lifetime and pulsed laser photon absorption experiments, Appl. Phys. 22, 273 (1980).

[21] M. Deutsch, Three-quantum decay of positronium, Phys. Rev. 83, 866 (1951).

[22] B. S. Cooper, A. M. Alonso, A. Deller, T. E. Wall, and D. B. Cassidy, A trap-based pulsed positron beam optimised for positronium laser spectroscopy, Rev. Sci. Instrum. 86, 103101 (2015).

[23] A. P. Mills, Jr., Experiments with dense low-energy positrons and positronium, Adv. At. Mol. Opt. Phys. 65, 265 (2016).

[24] T. F. Gallagher, Rydberg Atoms (Cambridge University Press, Cambridge, 1994).

[25] K. P. Ziock, R. H. Howell, F. Magnotta, R. A. Failor, and K. M. Jones, First Observation of Resonant Excitation of High$n$ States in Positronium, Phys. Rev. Lett. 64, 2366 (1990).

[26] D. B. Cassidy, T. H. Hisakado, H. W. K. Tom, and A. P. Mills, Jr., Efficient Production of Rydberg Positronium, Phys. Rev. Lett. 108, 043401 (2012).

[27] A. M. Alonso, B. S. Cooper, A. Deller, S. D. Hogan, and D. B. Cassidy, Controlling Positronium Annihilation with Electric Fields, Phys. Rev. Lett. 115, 183401 (2015). 
[28] A. Deller, A. M. Alonso, B. S. Cooper, S. D. Hogan, and D. B. Cassidy, Measurement of Rydberg positronium fluorescence lifetimes, Phys. Rev. A 93, 062513 (2016).

[29] S. D. Hogan, Rydberg-Stark deceleration of atoms and molecules, EPJ Tech. Instrum. 3, 1 (2016).

[30] W. H. Wing, Electrostatic Trapping of Neutral Atomic Particles, Phys. Rev. Lett. 45, 631 (1980).

[31] T. Breeden and H. Metcalf, Stark Acceleration of Rydberg Atoms in Inhomogeneous Electric Fields, Phys. Rev. Lett. 47, 1726 (1981).

[32] D. Townsend, A. L. Goodgame, S. R. Procter, S. R. Mackenzie, and T. P. Softley, Deflection of krypton Rydberg atoms in the field of an electric dipole, J. Phys. B 34, 439 (2001).

[33] Y. Yamakita, S. R. Procter, A. L. Goodgame, T. P. Softley, and F. Merkt, Deflection and deceleration of hydrogen Rydberg molecules in inhomogeneous electric fields, J. Chem. Phys. 121, 1419 (2004).

[34] E. Vliegen and F. Merkt, On the electrostatic deceleration of argon atoms in high Rydberg states by time-dependent inhomogeneous electric fields, J. Phys. B 38, 1623 (2005).

[35] E. Vliegen, P. A. Limacher, and F. Merkt, Measurement of the three-dimensional velocity distribution of Stark-decelerated Rydberg atoms, Eur. Phys. J. D 40, 73 (2006).

[36] E. Vliegen, H. J. Wörner, T. P. Softley, and F. Merkt, Nonhydrogenic Effects in the Deceleration of Rydberg Atoms in Inhomogeneous Electric Fields, Phys. Rev. Lett. 92, 033005 (2004).

[37] E. Vliegen and F. Merkt, Normal-Incidence Electrostatic Rydberg Atom Mirror, Phys. Rev. Lett. 97, 033002 (2006).

[38] P. Lancuba and S. D. Hogan, Guiding Rydberg atoms above surface-based transmission lines, Phys. Rev. A 88, 043427 (2013).

[39] P. Allmendinger, J. Deiglmayr, J. A. Agner, H. Schmutz, and F. Merkt, Surface-electrode decelerator and deflector for Rydberg atoms and molecules, Phys. Rev. A 90, 043403 (2014).

[40] E. Vliegen, S. D. Hogan, H. Schmutz, and F. Merkt, Stark deceleration and trapping of hydrogen Rydberg atoms, Phys. Rev. A 76, 023405 (2007).

[41] S. D. Hogan and F. Merkt, Demonstration of ThreeDimensional Electrostatic Trapping of State-Selected Rydberg Atoms, Phys. Rev. Lett. 100, 043001 (2008).

[42] Ch. Seiler, S. D. Hogan, H. Schmutz, J. A. Agner, and F. Merkt, Collisional and Radiative Processes in Adiabatic Deceleration, Deflection and Off-Axis Trapping of a Rydberg Atom Beam, Phys. Rev. Lett. 106, 073003 (2011).

[43] S. D. Hogan, P. Allmendinger, H. Saßmannshausen, H. Schmutz, and F. Merkt, Surface-Electrode Rydberg-Stark Decelerator, Phys. Rev. Lett. 108, 063008 (2012).

[44] P. Lancuba and S. D. Hogan, Transmission-line decelerators for atoms in high Rydberg states, Phys. Rev. A 90, 053420 (2014).

[45] P. Lancuba and S. D. Hogan, Electrostatic trapping and in situ detection of Rydberg atoms above chip-based transmission lines, J. Phys. B 49, 074006 (2016).

[46] S. D. Hogan, Ch. Seiler, and F. Merkt, Motional, isotope and quadratic Stark effects in Rydberg-Stark deceleration and off-axis electric trapping of H and D, J. Phys. B 46, 045303 (2013).

[47] Ch. Seiler, J. A. Agner, P. Pillet, and F. Merkt, Radiative and collisional processes in translationally cold samples of hydrogen Rydberg atoms studied in an electrostatic trap, J. Phys. B 49, 094006 (2016).

[48] S. D. Hogan, Ch. Seiler, and F. Merkt, Rydberg-State-Enabled Deceleration and Trapping of Cold Molecules, Phys. Rev. Lett. 103, 123001 (2009).

[49] Ch. Seiler, S. D. Hogan, and F. Merkt, Trapping cold molecular hydrogen, Phys. Chem. Chem. Phys. 13, 19000 (2011).

[50] P. Allmendinger, J. Deiglmayr, O. Schullian, K. Höveler, J. A. Agner, H. Schmutz, and F. Merkt, New method to study ion-molecule reactions at low temperatures and application to the $\mathrm{H}_{2}^{+}+\mathrm{H}_{2} \longrightarrow \mathrm{H}_{3}^{+}+\mathrm{H}$ reaction, ChemPhysChem 17, 3596 (2016).

[51] P. Allmendinger, J. Deiglmayr, K. Höveler, O. Schullian, and F. Merkt, Observation of enhanced rate coefficients in the $\mathrm{H}_{2}^{+}+$ $\mathrm{H}_{2} \longrightarrow \mathrm{H}_{3}^{+}+\mathrm{H}$ reaction at low collision energies, J. Chem. Phys. 145, 244316 (2016).

[52] A. Deller, A. M. Alonso, B. S. Cooper, S. D. Hogan, and D. B. Cassidy, Electrostatically Guided Rydberg Positronium, Phys. Rev. Lett. 117, 073202 (2016).

[53] S. A. Rangwala, T. Junglen, T. Rieger, P. W. H. Pinkse, and G. Rempe, Continuous source of translationally cold dipolar molecules, Phys. Rev. A 67, 043406 (2003).

[54] M. Motsch, L. D. van Buuren, C. Sommer, M. Zeppenfeld, G. Rempe, and P. W. H. Pinkse, Cold guided beams of water isotopologs, Phys. Rev. A 79, 013405 (2009).

[55] L. D. van Buuren, C. Sommer, M. Motsch, S. Pohle, M. Schenk, J. Bayerl, P. W. H. Pinkse, and G. Rempe, Electrostatic Extraction of Cold Molecules from a Cryogenic Reservoir, Phys. Rev. Lett. 102, 033001 (2009).

[56] B. Bertsche and A. Osterwalder, State-selective detection of velocity-filtered $\mathrm{ND}_{3}$ molecules, Phys. Rev. A 82, 033418 (2010).

[57] C. Sommer, M. Motsch, S. Chervenkov, L. D. van Buuren, M. Zeppenfeld, P. W. H. Pinkse, and G. Rempe, Velocity-selected molecular pulses produced by an electric guide, Phys. Rev. A 82, 013410 (2010).

[58] A. C. L. Jones, H. J. Rutbeck-Goldman, T. H. Hisakado, A. M. Piñeiro, H. W. K. Tom, A. P. Mills, B. Barbiellini, and J. Kuriplach, Angle-Resolved Spectroscopy of Positronium Emission from a Cu(110) Surface, Phys. Rev. Lett. 117, 216402 (2016).

[59] A. P. Mills, Jr. and M. Leventhal, Can we measure the gravitational free fall of cold Rydberg state positronium? Nucl. Instrum. Methods Phys. Res., Sect. B 192, 102 (2002).

[60] D. B. Cassidy and S. D. Hogan, Atom control and gravity measurements using Rydberg positronium, Int. J. Mod. Phys. Conf. Ser. 30, 1460259 (2014).

[61] F. Merkt and R. N. Zare, On the lifetimes of Rydberg states probed by delayed pulsed field ionization, J. Chem. Phys. 101, 3495 (1994).

[62] A C. L. Jones, T. H. Hisakado, H. J. Goldman, H. W. K. Tom, and A. P. Mills, Jr., Polarization dependence of $n=2$ positronium transition rates to Stark-split $n=30$ levels via crossed-beam spectroscopy, J. Phys. B 49, 064006 (2016).

[63] R. J. Damburg and V. V. Kolosov, Theoretical studies of hydrogen Rydberg atoms in electric fields, in Rydberg States of Atoms and Molecules, edited by R. F. Stebbings and F. B. Dunning (Cambridge University Press, Cambridge, 1983), pp. 31-72. 
[64] S. D. Hogan, Calculated photoexcitation spectra of positronium Rydberg states, Phys. Rev. A 87, 063423 (2013).

[65] A. M. Alonso, B. S. Cooper, A. Deller, and D. B. Cassidy, Single-shot positron annihilation lifetime spectroscopy with LYSO scintillators, Nucl. Instrum. Methods Phys. Res., Sect. A 828, 163 (2016).

[66] L. Liszkay, M. F. Barthe, C. Corbel, P. Crivelli, P. Desgardin, M. Etienne, T. Ohdaira, P. Perez, R. Suzuki, V. Valtchev, and A. Walcarius, Orthopositronium annihilation and emission in mesostructured thin silica and silicalite-1 films, Appl. Surf. Sci. 255, 187 (2008).

[67] L. Liszkay, F. Guillemot, C. Corbel, J.-P. Boilot, T. Gacoin, E. Barthel, P. Prez, M.-F. Barthe, P. Desgardin, P. Crivelli, U. Gendotti, and A. Rubbia, Positron annihilation in latextemplated macroporous silica films: Pore size and orthopositronium escape, New J. Phys. 14, 065009 (2012).

[68] T. E. Wall, A. M. Alonso, B. S. Cooper, A. Deller, S. D. Hogan, and D. B. Cassidy, Selective Production of RydbergStark States of Positronium, Phys. Rev. Lett. 114, 173001 (2015).

[69] D. B. Cassidy and A. P. Mills, Jr., A fast detector for singleshot positron annihilation lifetime spectroscopy, Nucl. Instrum. Methods Phys. Res., Sect. A 580, 1338 (2007).

[70] D. B. Cassidy, S. H. M. Deng, H. K. M. Tanaka, and A. P. Mills, Jr., Single shot positron annihilation lifetime spectroscopy, Appl. Phys. Lett. 88, 194105 (2006).

[71] C. G. Fischer, M. H. Weber, C. L. Wang, S. P. McNeil, and K. G. Lynn, Positronium in low temperature mesoporous films, Phys. Rev. B 71, 180102 (2005).

[72] D. W. Gidley, H. G. Peng, and R. S. Vallery, Positron annihilation as a method to characterize porous materials, Annu. Rev. Mater. Res. 36, 49 (2006).

[73] L. Liszkay, C. Corbel, P. Perez, P. Desgardin, M. F. Barthe, T. Ohdaira, R. Suzuki, P. Crivelli, U. Gendotti, A. Rubbia, M. Etienne, and A. Walcarius, Positronium reemission yield from mesostructured silica films, Appl. Phys. Lett. 92, 063114 (2008).

[74] D. B. Cassidy, P. Crivelli, T. H. Hisakado, L. Liszkay, V. E. Meligne, P. Perez, H. W. K. Tom, and A. P. Mills, Jr., Positronium cooling in porous silica measured via Doppler spectroscopy, Phys. Rev. A 81, 012715 (2010).

[75] D. B. Cassidy, T. H. Hisakado, V. E. Meligne, H. W. K. Tom, and A. P. Mills, Jr., Delayed emission of cold positronium from mesoporous materials, Phys. Rev. A 82, 052511 (2010).

[76] P. Crivelli, U. Gendotti, A. Rubbia, L. Liszkay, P. Perez, and C. Corbel, Measurement of the orthopositronium confinement energy in mesoporous thin films, Phys. Rev. A 81, 052703 (2010).

[77] C. He, S. Wang, Y. Kobayashi, T. Ohdaira, and R. Suzuki, Role of pore morphology in positronium diffusion in mesoporous silica thin films and in positronium emission from the surfaces, Phys. Rev. B 86, 075415 (2012).

[78] Y. Zhou, W. Mao, Q. Li, J. Wang, and C. He, Formation and annihilation of positronium in silica aerogels under atmosphere of oxygen and nitrogen mixture, Chem. Phys. 459, 81 (2015).

[79] A. Deller, B. S. Cooper, T. E. Wall, and D. B. Cassidy, Positronium emission from mesoporous silica studied by laserenhanced time-of-flight spectroscopy, New J. Phys. 17, 043059 (2015).
[80] B. S. Cooper, A. M. Alonso, A. Deller, L. Liszkay, and D. B. Cassidy, Positronium production in cryogenic environments, Phys. Rev. B 93, 125305 (2016).

[81] A. M. Alonso, S. D. Hogan, and D. B. Cassidy, Production of $2^{3} S_{1}$ positronium atoms by single-photon excitation in an electric field, Phys. Rev. A 95, 033408 (2017).

[82] A. R. Swann, D. B. Cassidy, A. Deller, and G. F. Gribakin, Formation of positron-atom bound states in collisions between Rydberg Ps and neutral atoms, Phys. Rev. A 93, 052712 (2016).

[83] J. C. Greenbank and B. B. Argent, Vapour pressure of magnesium, zinc and cadmium, Trans. Faraday Soc. 61, 655 (1965).

[84] E. K. Anderson, R. A. Boadle, J. R. Machacek, L. Chiari, C. Makochekanwa, S. J. Buckman, M. J. Brunger, G. Garcia, F. Blanco, O. Ingolfsson, and J. P. Sullivan, Low energy positron interactions with uracil-total scattering, positronium formation, and differential elastic scattering cross sections, J. Chem. Phys. 141, 034306 (2014).

[85] B. Ghaffari, J. M. Gerton, W. I. McAlexander, K. E. Strecker, D. M. Homan, and R. G. Hulet, Laser-free slow atom source, Phys. Rev. A 60, 3878 (1999).

[86] A. C. L. Jones, T. H. Hisakado, H. J. Goldman, H. W. K. Tom, A. P. Mills, Jr., and D. B. Cassidy, Doppler-corrected Balmer spectroscopy of Rydberg positronium, Phys. Rev. A 90, 012503 (2014).

[87] A. C. L. Jones, A. M. Piñeiro, E. E. Roeder, H. J. RutbeckGoldman, H. W. K. Tom, and A. P. Mills, Jr., Large-area fieldionization detector for the study of Rydberg atoms, Rev. Sci. Instrum. 87, 113307 (2016).

[88] R. Paulin and G. Ambrosino, Annihilation libre de l'orthopositonium formé dans certaines poudres de grande surface spécifique, J. Phys. (Paris) 29, 263 (1968).

[89] K. F. Canter, A. P. Mills, Jr., and S. Berko, Efficient Positronium Formation by Slow Positrons Incident on Solid Targets, Phys. Rev. Lett. 33, 7 (1974).

[90] Y. Nagashima, Y. Morinaka, T. Kurihara, Y. Nagai, T. Hyodo, T. Shidara, and K. Nakahara, Origins of positronium emitted from $\mathrm{SiO}_{2}$, Phys. Rev. B 58, 12676 (1998).

[91] D. B. Cassidy, T. H. Hisakado, H. W. K. Tom, and A. P. Mills, Jr., New Mechanism for Positronium Formation on a Silicon Surface, Phys. Rev. Lett. 106, 133401 (2011).

[92] D. Dutta, J. I. Feldblyum, D. W. Gidley, J. Imirzian, M. Liu, A. J. Matzger, R. S. Vallery, and A. G. Wong-Foy, Evidence of Positronium Bloch States in Porous Crystals of $\mathrm{Zn}_{4}$ O-Coordination Polymers, Phys. Rev. Lett. 110, 197403 (2013).

[93] M. Charlton, Experimental studies of positrons scattering in gases, Rep. Prog. Phys. 48, 737 (1985).

[94] P. Caradonna, A. Jones, C. Makochekanwa, D. S. Slaughter, J. P. Sullivan, S. J. Buckman, I. Bray, and D. V. Fursa, Highresolution positron scattering from helium: Grand total and positronium-formation cross sections, Phys. Rev. A 80, 032710 (2009).

[95] A. P. Mills, Jr. and L. Pfeiffer, Desorption of Surface Positrons: A Source of Free Positronium at Thermal Velocities, Phys. Rev. Lett. 43, 1961 (1979).

[96] A. P. Mills, Jr., Thermal activation measurement of positron binding energies at surfaces, Solid State Commun. 31, 623 (1979). 
[97] S. Mariazzi, P. Bettotti, and R. S. Brusa, Positronium Cooling and Emission in Vacuum from Nanochannels at Cryogenic Temperature, Phys. Rev. Lett. 104, 243401 (2010).

[98] A. P. Mills, E. D. Shaw, M. Leventhal, R. J. Chichester, and D. M. Zuckerman, Thermal desorption of cold positronium from oxygen-treated Al(111) surfaces, Phys. Rev. B 44, 5791 (1991).

[99] J. Mitroy, M. W. J. Bromley, and G. G. Ryzhikh, Positron and positronium binding to atoms, J. Phys. B 35, R81 (2002).

[100] X. Cheng, D. Babikov, and D. M. Schrader, Binding-energy predictions of positrons and atoms, Phys. Rev. A 83, 032504 (2011).

[101] C. Harabati, V. A. Dzuba, and V. V. Flambaum, Identification of atoms that can bind positrons, Phys. Rev. A 89, 022517 (2014).

[102] J. R. Machacek, R. Boadle, S. J. Buckman, and J. P. Sullivan, Search for positron quasibound states in the doubly excited region of the helium atom, Phys. Rev. A 86, 064702 (2012).

[103] G. F. Gribakin, J. A. Young, and C. M. Surko, Positronmolecule interactions: Resonant attachment, annihilation, and bound states, Rev. Mod. Phys. 82, 2557 (2010).

[104] J. Mitroy and G. G. Ryzhikh, Measuring the positron affinities of atoms, J. Phys. B 32, L411 (1999).

[105] C. M. Surko, J. R. Danielson, G. F. Gribakin, and R. E. Continetti, Measuring positron-atom binding energies through laser-assisted photorecombination, New J. Phys. 14, 065004 (2012).

[106] H. Hotop and A. Niehaus, Reactions of excited atoms and molecules with atoms and molecules, Z. Phys. 215, 395 (1968).

[107] W. P. West, G. W. Foltz, F. B. Dunning, C. J. Latimer, and R. F. Stebbings, Absolute Measurements of Collisional Ionization of Xenon Atoms in Well-Defined High Rydberg States, Phys. Rev. Lett. 36, 854 (1976).

[108] S. J. Brawley, S. Armitage, J. Beale, D. E. Leslie, A. I. Williams, and G. Laricchia, Electron-like scattering of positronium, Science 330, 789 (2010).

[109] S. J. Brawley, A. I. Williams, M. Shipman, and G. Laricchia, Resonant Scattering of Positronium in Collision with $\mathrm{CO}_{2}$, Phys. Rev. Lett. 105, 263401 (2010).

[110] G. F. Chew and G. C. Wick, The impulse approximation, Phys. Rev. 85, 636 (1952).

[111] I. I. Fabrikant and G. F. Gribakin, Similarity between Positronium-Atom and Electron-Atom Scattering, Phys. Rev. Lett. 112, 243201 (2014).
[112] J. P. Merrison, H. Bluhme, J. Chevallier, B. I. Deutch, P. Hvelplund, L. V. Jørgensen, H. Knudsen, M. R. Poulsen, and M. Charlton, Hydrogen Formation by Proton Impact on Positronium, Phys. Rev. Lett. 78, 2728 (1997).

[113] S. Willitsch, M. T. Bell, A. D. Gingell, S. R. Procter, and T. P. Softley, Cold Reactive Collisions between Laser-Cooled Ions and Velocity-Selected Neutral Molecules, Phys. Rev. Lett. 100, 043203 (2008).

[114] B. C. Sawyer, B. K. Stuhl, M. Yeo, T. V. Tscherbul, M. T. Hummon, Y. Xia, J. Klos, D. Patterson, J. M. Doyle, and J. Ye, Cold heteromolecular dipolar collisions, Phys. Chem. Chem. Phys. 13, 19059 (2011).

[115] M. Charlton, Antihydrogen production in collisions of antiprotons with excited states of positronium, Phys. Lett. A 143, 143 (1990).

[116] P. Pérez, D. Banerjee, F. Biraben, D. Brook-Roberge, M. Charlton, P. Cladé, P. Comini, P. Crivelli, O. Dalkarov, P. Debu, A. Douillet, G. Dufour, P. Dupré, S. Eriksson, P. Froelich, P. Grandemange, S. Guellati, R. Guérout, J. M. Heinrich, P.-A. Hervieux et al., The GBAR antimatter gravity experiment, Hyperfine Interact. 233, 21 (2015).

[117] M. Doser, C. Amsler, A. Belov, G. Bonomi, P. Brunig, J. Bremer, R. Brusa, G. Burkhart, L. Cabaret, C. Canali, F. Castelli, K. Chlouba, S. Cialdi, D. Comparat, G. Consolati, L. Di. Noto, A. Donzella, A. Dudarev, T. Eisel, R. Ferragut et al. (AEGIS Collaboration), Exploring the WEP with a pulsed cold beam of antihydrogen, Classical Quantum Gravity 29, 184009 (2012).

[118] A. S. Kadyrov, C. M. Rawlins, A. T. Stelbovics, I. Bray, and M. Charlton, Antihydrogen Formation via Antiproton Scattering with Excited Positronium, Phys. Rev. Lett. 114, 183201 (2015).

[119] C. M. Rawlins, A. S. Kadyrov, A. T. Stelbovics, I. Bray, and M. Charlton, Calculation of antihydrogen formation via antiproton scattering with excited positronium, Phys. Rev. A 93, 012709 (2016).

[120] A. Osterwalder and F. Merkt, Using High Rydberg States as Electric Field Sensors, Phys. Rev. Lett. 82, 1831 (1999).

[121] R. Pohl, A. Antognini, F. Nez, F. D. Amaro, F. Biraben, J. M. R. Cardoso, D. S. Covita, A. Dax, S. Dhawan, L. M. P. Fernandes, A. Giesen, T. Graf, T. W. Hänsch, P. Indelicato, L. Julien, C.-Y. Kao, P. Knowles, E.-O. Le Bigot, Y.-W. Liu, A. M. Lopes et al., The size of the proton, Nature (London) 466, 213 (2010). 